\title{
Leptin and leptin receptor gene polymorphisms and obstructive sleep apnea syndrome: is there an association?
}

\author{
Sophia E. Schiza • Charalampos Mermigkis • \\ Izolde Bouloukaki
}

Received: 21 January 2015 /Revised: 28 January 2015 / Accepted: 30 January 2015 /Published online: 11 February 2015

(C) Springer-Verlag Berlin Heidelberg 2015

Obstructive sleep apnea syndrome (OSAS) is a common chronic disorder that often requires lifelong care. Although the pathogenetic mechanism of OSAS remains unclear, a combination of multiple gene interactions and environmental factors has been incriminated. It has been reported that genetic factors could account for about $40 \%$ of the variance in the apnea-hypopnea index (AHI) of OSAS patients [1]. Genes might increase susceptibility of OSAS through at least four primary intermediate pathogenic pathways: obesity and related metabolic phenotypes, craniofacial and upper airway morphology, control of ventilation and control of sleep and circadian rhythm. On the basis of this conception, researchers have sought to identify genetic variants that determine these intermediate phenotypes for OSAHS. These variants could potentially contribute to earlier recognition and treatment, as well as identification of potential targets for novel therapies.

Among candidate genes affecting the risk of OSAS, there are genes whose polymorphisms influence the development of diseases with similar pathogenesis to OSAS. Owing to the strong association between obesity and OSAS, leptin genes have been proposed as candidate genes for OSAS [2]. Leptin plays an important role not only in the regulation of body weight but also in exerting a number of other effects relevant to OSAS, such as lung growth, respiratory control, and sleep architecture. Leptin acts through the leptin receptor (LEPR), a single-transmembrane-domain receptor of the cytokine recep-

S. E. Schiza $(\triangle) \cdot$ C. Mermigkis $\cdot$ I. Bouloukaki

Sleep Disorders Center, Department of Thoracic Medicine,

University General Hospital, Medical School of the University of

Crete, 71110 Heraklion, Crete, Greece

e-mail: schiza@med.uoc.gr tor family, which is found in many tissues in several alternatively spliced forms. There are several common variants of the leptin and LEPR genes, and the potential associations of these variants with obesity have been evaluated in different populations [3-5].

In this issue of Sleep and Breathing, Dr. Lv and colleagues conducted a systematic review of English and Chinese published studies investigating the role of leptin and LEPR polymorphisms in OSAS and performed a meta-analysis of these studies. The principal finding based on the findings of seven small sample publications was to confirm that there is no association between OSAS and leptin and LEPR polymorphisms. However, another observation made in this study was that in European, but not in Asian population, Gln233Arg polymorphism was associated with a low risk for OSAS. Although the authors have used an appropriate study design, their results should be interpreted with caution as the number of included studies was relatively small, restricting the power of the study and not allowing subgroup meta-analysis. Furthermore, as the author explain the inclusion of only English and Chinese language studies and the exclusion of unpublished and missing studies from the literature review could have contributed to publication bias. Therefore, more suitable studies with adequate sample sizes should be employed in order to further research into the genetic origins of OSAS in this area.

In the past years, a growing number of studies has examined the role of leptin in the respiratory system, showing that leptin regulation is altered in OSAS [6]. However, there is a large variation of leptin concentrations as well as changes in OSAS groups; the response of leptin concentrations to CPAP treatment also has some inconsistency [7]. Furthermore, the potential associations of leptin and LEPR gene polymorphisms with OSAS have been assessed in different 
populations with inconsistent results [8-10]. Inadequate study design, small samples, and confounding variables like difference in ethnicity of samples, age, sex, and obesity, the most important among them, represent a significant challenge in design of valid and reproducible studies. In addition, studies so far could not evaluate gene-gene and gene-environment interactions due to the small number of patients identified.

Nevertheless, one should keep in mind that OSAS is a multifactorial disease and might not be determined by a single gene mutation, but from a combination of susceptibility genes and environmental factors. Therefore, various factors other than gene structure may set off or facilitate disease progression in the course of time. Despite that, knowledge of genetic risk factors is essential to develop predictive models that incorporate both genetic and phenotypic markers thereby enabling early diagnosis and appropriate interventions. Unfortunately, there are many unanswered questions about OSAS and leptin and much remains to be done on the genetics of OSAHS.

In conclusion, the association between leptin and leptin receptor gene polymorphisms and susceptibility to OSAS remains poorly defined due conflicting data. Although significant progress has been made in identifying the genetic basis of sleep disorders such as restless leg syndrome and narcolepsy, the genetic basis of OSAS remains to be determined. This review by Dr. Lv et al. definitely has clinical importance as knowledge of leptin genetic variants associated with OSAS expands our understanding of the genetic background of OSAS and holds promise as a therapeutic option. Still, these findings warrant further investigations and trials conducted in large patient populations. Future research should also focus on the clinical relevance of these findings with the ultimate goal of reducing morbidity and public health concerns associated with OSAHS.

\section{References}

1. Redline S, Tishler PV, Tosteson TD, Williamson J, Kump K, Browner I, Ferrette V, Krejci P (1995) The familial aggregation of obstructive sleep apnea. Am J Respir Crit Care Med 151:682-687

2. Patel SR (2005) Shared genetic risk factors for obstructive sleep apnea and obesity. J Appl Physiol 99:1600-1606

3. Yiannakouris N, Yannakoulia M, Melistas L, Chan JL, Klimis-Zacas D, Mantzoros CS (2001) The Q223R polymorphism of the leptin receptor gene is significantly associated with obesity and predicts a small percentage of body weight and body composition variability. $\mathrm{J}$ Clin Endocrinol Metab 86:4434-4439

4. Shintani M, Ikegami H, Yamato E, Kawaguchi Y, Fujisawa T, Nakagawa Y, Hamada Y, Ueda H, Miki T, Ogihara T (1996) A novel microsatellite polymorphism in the human OB gene: a highly polymorphic marker for linkage analysis. Diabetologia 39:1398-1401

5. Matsuoka N, Ogawa Y, Hosoda K, Matsuda J, Masuzaki H, Miyawaki T, Azuma N, Natsui K, Nishimura H, Yoshimasa Y, Nishi S, Thompson DB, Nakao K (1997) Human leptin receptor gene in obese Japanese subjects: evidence against either obesity-causing mutations or association of sequence variants with obesity. Diabetologia 40:1204-1210

6. Malli F, Papaioannou AI, Gourgoulianis KI, Daniil Z (2010) The role of leptin in the respiratory system: an overview. Respir Res 11:152

7. Pan W, Kastin AJ (2014) Leptin: a biomarker for sleep disorders? Sleep Med Rev 18:283-290

8. Popko K, Gorska E, Wasik M, Plywaczewski R, Winiarska M, Gorecka D, Sliwinski P, Demkow U (2007) Frequency of distribution of leptin receptor gene polymorphism in obstructive sleep apnea patients. J Physiol Pharmacol 58(Suppl 5):551-561

9. Hanaoka M, Yu X, Urushihata K, Ota M, Fujimoto K, Kubo KM (2008) Leptin and leptin receptor gene polymorphisms in obstructive sleep apnea syndrome. Chest 133:79-85

10. Huang R, Huang X, Li M, Xiao Y, Zhang J (2003) An investigation of the relationship between Lepr gene Gln223Arg polymorphism and obstructive sleep apnea hypopnea syndrome. Chin J Tuberc Respir Dis 26:517-521 